\title{
Integration of Habitat Grammars for Biodiverse and Resilient Coastal Structures
}

\author{
Keith Van de Riet, Jessene Aquino-Thomas, \\ Pieter Conradie
}

ABSTRACT - Contemporary architectural ornament serves multiple purposes; articulated surfaces are no longer simply symbolic gesture but, in addition, contain material performance as expression of technological, environmental and other cultural factors. At the same time, parametric design has opened new frontiers in patterning and bio-inspired design. The manifestation of these bio-inspired materials can be compelling spatial narratives that speculate on the blending of nature and architecture. However, these biophilic tendencies might further serve in the development of more sustainable architecture and urbanism; surfaces and structures derived from nature might also serve nature.

In this work, we present a method to define "habitat grammars" that create functional spaces for diverse species, as well as generating unique aesthetic properties derived from regional nuances in flora and fauna. The method is applied to a seawall in South Florida near mangroves. Panels were cast in high strength concrete and experimentally observed in aquariums with live specimens. The results indicated that low relief panels performed equivalent to featureless walls, whereas higher relief panels elicited a near unanimous response from the introduced species.

Keywords: artificial mangrove, biomimicry, constructed habitat, engineered-living, habitat grammars

Architectural ornament became catalogued for designers during the nineteenth and early twentieth centuries. Many professionals of the era were referencing these manuals to incorporate samples from regional 
aesthetics of the world. These architectural expressions contained a plethora of symbolic, mythological and more prosaic references that were used to distinguish the architect and developer by referencing patterns and symbols fashionable of the time. Today's ornament serves another purpose; the articulation of surfaces is no longer an abstract representation, but rather contains material performance in place of gilded symbols. Parallel to this, parametric design has opened new frontiers in material patterning and bio-inspired design within constructed landscapes. Increasingly popular are patterns derived from nature using basic image sampling techniques. The manifestation of these bio-inspired materials can be compelling spatial narratives that speculate on the blending of nature and architecture. However, these biophilic tendencies might further serve in the development of more sustainable architecture and urbanism; surfaces and structures derived from nature might also serve nature.

Photographic evidence of natural environments is often a source of inspiration for patterns of structure and materials in design. Even in the most basic application, these photo-derived architectural effects contribute to the overall play of material, form and light in buildings. Computational technologies have made the integration of natural patterns for design purposes relatively simple; image sampling and its derivatives are readily available in most modeling software in various forms and can be easily incorporated into design workflow for organic pattern creation. The surfaces generated from this procedure are manipulated with parameters that control the sampling density of the image, as well as the translation of pixel color values into spatial conditions driven by vectors or other transformation. The resulting surfaces and geometries create appealing material effects useful in the design of architectural materials.

In the case of architectural design, these sampled patterns are driven by human requirements of thermal performance (shading), privacy (screens), aesthetics and other criteria. The images and their derivations can be manually interpreted by cropping, scaling and modifying them to fit with needs or desires, akin to other creative activities that blend or mix patterns, such as music sampling (Goldman and Zdepski 1990). Compositions can be as simple as translating an image to surface pattern as perforation, etching, etc. or rather complex transformations that capture natural phenomena with patterns that dissolve the image through experimental material and fabrication processes that recreate environmental effects (Fig. 1). Adapted further, image sampling of natural patterns may serve as a method to bridge between engineered structures and living systems to support diverse forms of life. This technique could be used to capture and make available for design the characteristics of living and non-living systems from selected habitats.

This project utilizes a method for integrating habitat structure into architectural, infrastructural and landscape systems by combining image sampling with parametric models to generate panels that mimic natural 
habitats. We focus this approach on the context of South Florida's coastal urban landscapes to address the loss of tidal landscapes due to waterfront development.

\section{Coastal Urban Context: Human-altered Shorelines}

Within urban environments, the need for mutually beneficial connections between constructed and natural systems is becoming increasingly urgent. The majority of global population is now urban and located near coastlines and waterways, and these dense concentrations exert a disproportionate influence on environments and ecosystem function (Small 2004). Population density continues to grow in areas that contain fragile environments, and as a result, coastal ecosystems and their provisional services are in decline (Wells 2006). With regards to urban footprints near waterways, a rise in coastal armoring has resulted in a direct loss in natural shoreline, especially in foundation species. In some coastal cities, such as Sydney Australia, as much as $50 \%$ of the shoreline can be composed of coastal armor (Aguilera et al. 2014). In areas where there has been a loss of natural habitat there is a co-morbidity of a loss in biodiversity.

Many of the challenges to regenerate ecological systems stem from the fact that human environments replaced natural ones. For example, oyster extent declined by $64 \%$ (biomass reduced by $88 \%$ ) in U.S. coastal waters during the twentieth century (Ermgassen et al. 2012). Urbanization of waterfronts also has been shown to facilitate the spread of invasive species (Byrnes, Reynolds, and Stachowicz 2007; Sheehy and Vik 2010). Florida has over $11,000 \mathrm{mi}$. [17,700 km] of canals and waterways that intersect with urban areas (State of Florida 2017), much of which is lined with concrete seawalls that replaced tidal habitats and contributed to the decline of the
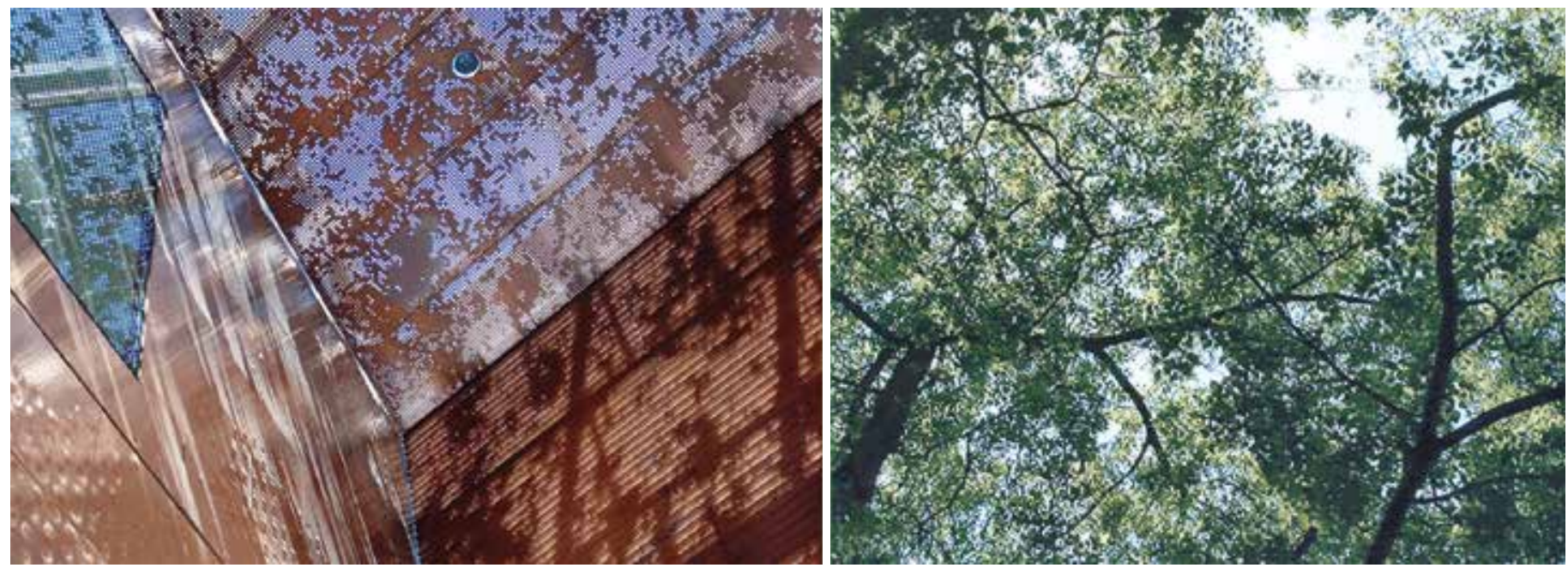

Figure 1. De Young Museum (Herzog \& de Meuron) perforated copper panels and forest canopy effect. 
environment (Figure 2). Mangroves and oysters are foundation species that are often removed or destroyed during shoreline armoring. Foundation species are habitat-modifying species that form the base of the community (Stachowicz 2001), have a disproportionately large effect on a community (Dayton 1972, Dayton 1975, Bruno \& Bertness 2001) and typically promote increases in diversity, resilience, food web complexity, and productivity. Within tidal environments of South Florida, diverse species occupy complex habitats, and structures that emulate natural mangrove and oyster shorelines could support the species and subspecies listed in Figure 2.

In terms of opportunity, studies have shown human-made structures have the potential to support diverse marine life and increase the filtration capacity of altered shorelines well beyond that of existing natural edges (Layman et al. 2014). "Seawalls, piles and other dock structures create surface area, and the choice of building material can play a substantial role in supporting tidal species (Chase et al., 2016; Theuerkauf et al., 2014)." "However, seawalls and other marine structures are relatively featureless vertical conditions and thus lack the structural complexity of natural intertidal habitats (Gittman et al., 2016)." Spatial heterogeneity allows for a more diverse species assemblage to occupy the habitat (Aguilera et al. 2014). For example, within the same ecosystem oysters on mangrove prop roots can be found in larger densities than on oyster reefs due to the additional vertical relief (Aquino-Thomas and Proffitt 2014), indicating that the design of a seawall to encourage oyster colonization can have cascading effects on water quality and biodiversity.

Although various performance metrics of a building can be simulated with a reasonable degree of certainty, the accommodation of living organisms and complex ecological processes requires a wide range of expertise. In many cases, there is a distinct gap in knowledge regarding dimensional preferences of species in these environments - many are adaptable to diverse habitat structures and documentation is not easily accomplished due to the discreet nature of the habitats. However, studies show supporting evidence that extending the available habitat across areas of human-altered landscapes would provide a simulacra of the historical natural shoreline. One survey found that replacement of large swaths of landscape (50-80\%) causes dramatic decline in species richness within human-altered shoreline environments, whereas fragmentation that permits patches of habitat to exist within proximity to each other (i.e. "habitat matrix") has little to no effect on most near shore marine species (Harwell, Posey, and Alphin 2011). Additionally, longer contiguous mangrove shorelines have been shown to increase the abundance of oysters on prop roots, which in turn facilitates oyster abundance on the ground (Aquino-Thomas and Proffitt 2014). Artificial habitat patches may provide a continuum across human interventions in order to promote diversity and improve water quality in these environments. 
Accommodating ecological services within urban areas has become paramount to reverse the decline of previously productive habitats, and the challenge may be one of design. Ecological engineering of artificial structures needs to be incorporated, particularly in areas where restoration efforts are unlikely to be implemented. Methods should not be limited to new structures, but should include methods to modify existing artificial structures.

Our collaborative research questions that guided the work include:

1. Can image sampling be appropriated for the creation of artificial habitats for non-human living systems within the constraints of a surface-based panel?

2. What effect does image composition (hue, saturation, grayscale, etc.) have on the image sampling results in terms of habitat structure and hierarchy?

3. Can the results of image sampling be effectively tuned for species and environments with secondary parametric inputs?

\section{METHODOLOGY}

Our method to creating artificial habitats utilizes image sampling coupled with parametric shape overlays and applies the approach in the design of panels for a living seawall in South Florida.
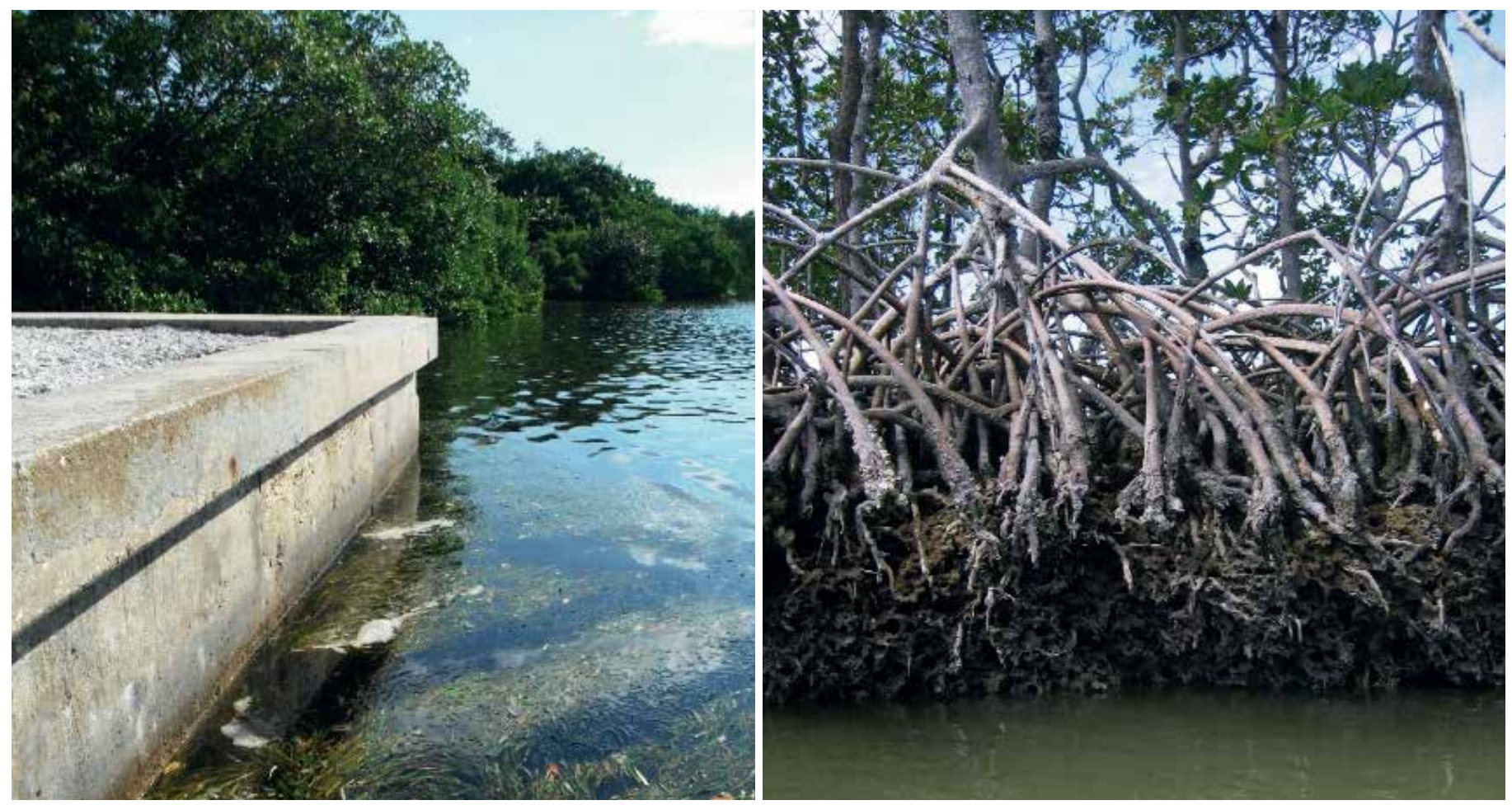

Figure 2. Sample "edge habitats" for South Florida, USA: (left) typical concrete seawall in Southwest Florida; (right) low tide view of mangrove-oyster reef in 10,000 Islands, Everglades National Park. 
Our methodology consisted of generating a wide range of surfaces derived from photographs of natural environments, then enhancing these images with specific geometry reflective of species habitat preferences - a set of shapes defined by habitat typologies for groups of tidal species.

We prioritized surface features that corresponded to evidence from extensive fieldwork undertaken by authors, accounting for the tool and material constraints of fabrication processes in the first generation of prototypes. Surfaces that scored best during interdisciplinary critique were digitally fabricated to establish fabrication and casting methods in preparation for aquarium-based experiments (not yet concluded at time of this publication).

\section{Image Sampling Parameters}

Our work utilized McNeel and Associates Rhinoceros modeling software with Grasshopper plug-in to create and modify the geometry. Originally, the "heightfield" command was used to sample images and modify parameters, but the limits of this tool led to the development of a custom script in Grasshopper. Our script generated the surface height by normalizing the image within a grayscale of 0 to 1 representing black to white. The final depth of panels ranged from 0 to $6 \mathrm{~cm}$ [2.36 in.] in $1,5 \mathrm{~cm}$ [0.59 in.] increments to simulate a range of depth approximating a flat seawall to the depth of relief in the outermost surface of an aggregated group of oysters. Images were selected based on variation in color and pattern and represented a range of habitat conditions in mangrove and oyster landscapes. These images were sampled and, in some cases, filters were applied to enhance image characteristics (contrast, saturation, hue, solarized, etc.) which maximized the diversity of topographical surfaces resulting from the sampling process. The parameters of the sampling procedure included density of sampling points (held constant in first series) and height of surface displacement, the combination of which represent the degree of fidelity to the original image.

\section{Parametric Shape Overlay}

We developed a script to generate primitive shape arrays that approximated basic habitat qualities for common species. Rather than target single species with each shape, the forms were meant to accommodate two types of habitat structures that serve many species - shelf and pocket forms. Shelves enable protected circulation and resting zones, while pockets serve as areas of refuge (Fig. 3). In the parametric model, circulation shelves are developed with rectangular bars defined by $\mathrm{x}$ and $\mathrm{y}$ inputs and the relief pockets are developed with a set of offset circles defined by radius inputs. In both cases, the shapes override points from the image sample array that lie within the bounds of the respective geometry - either to minimize or maximize the point height within the predefined limits of z-position. These two habitat typologies were intended as the first letters to a larger language of shapes that will be elaborated with the evolution of the system with feedback gained from the first prototype. 


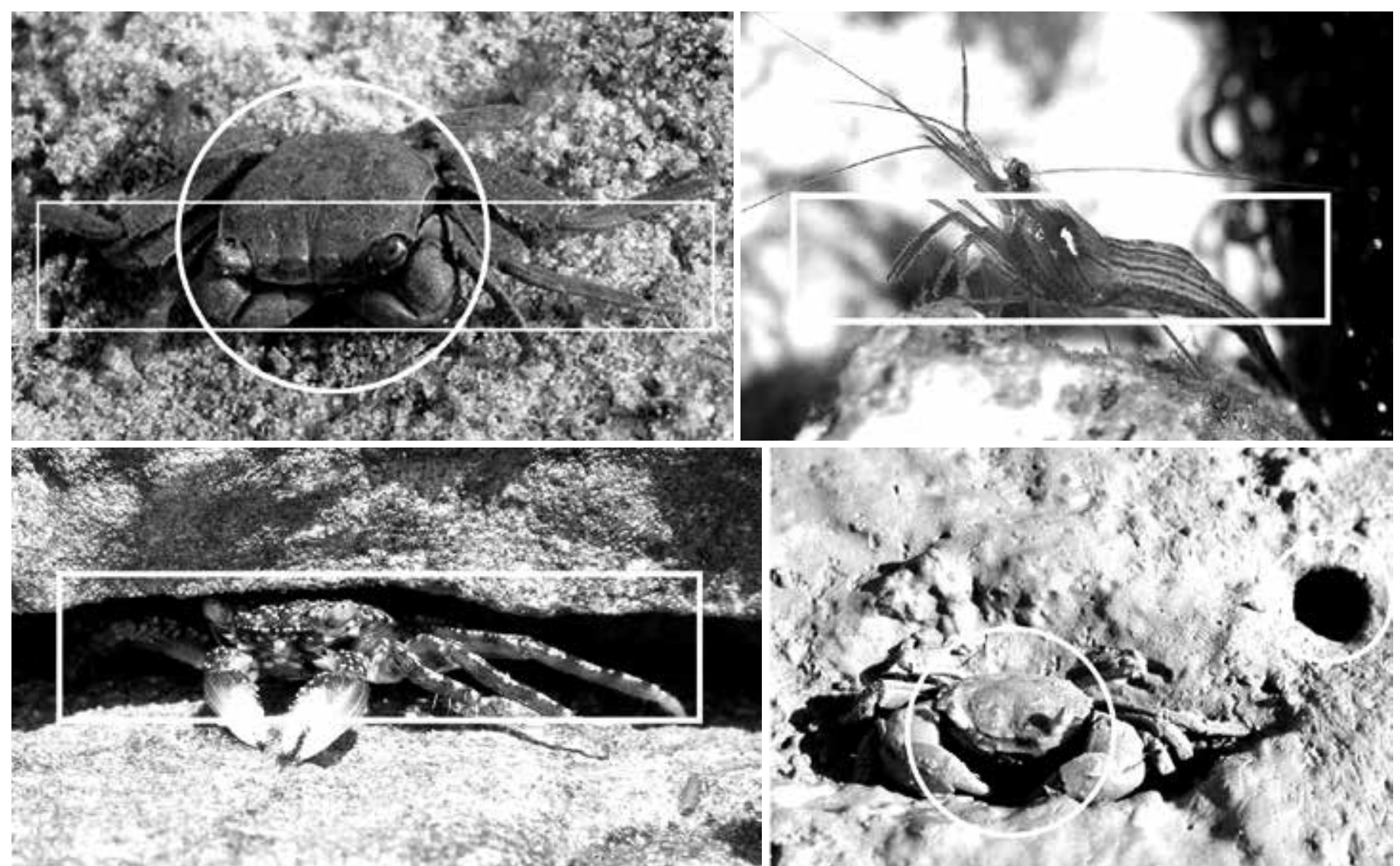

Figure 3. Shape definition by habitat type - shelf and pocket typologies satisfy a wide range of naturally-occurring habitats.

\section{RESULTS AND DISCUSSION}

Our work resulted in three specific outcomes: (a) a collaborative workflow and computational model able to synthesize criteria from multiple disciplines, (b) the evidence of this process that included a wide range of material samples and exploratory techniques, and (c) a final set of 20 panels that were cast in concrete for aquarium experiments relevant to South Florida seawall application.

We first relied on image sampling as an efficient method to produce a wide range of surface characteristics (Fig.4). Our database of images was established using author fieldwork and online sources. These images were edited by cropping, desaturating, inverting and applying color filters to manipulate the information and emphasize the distinct patterns. This first set of surfaces was driven by the images and maintained a constant surface offset height but varied density of sampling points from left to right across each set.

Most significantly, the diverse range of formal characteristics across the set of panels enabled critical evaluation of the results that ultimately led to the shape enhancement strategy. Due to the lack of documented habitat 


\section{딜}

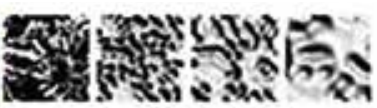
담

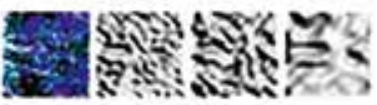
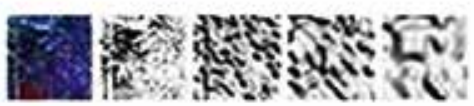

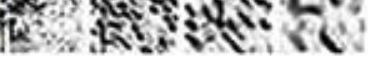
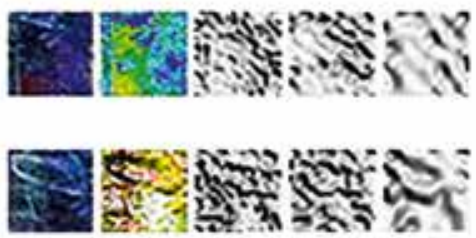

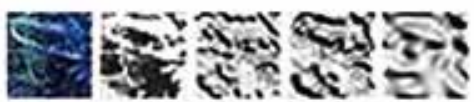
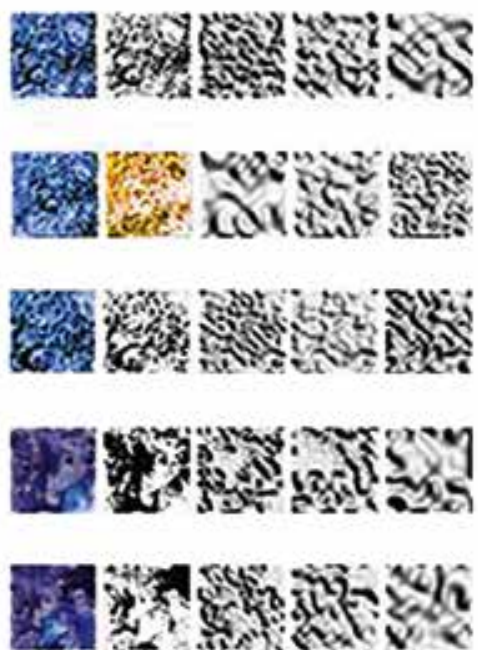

iscing
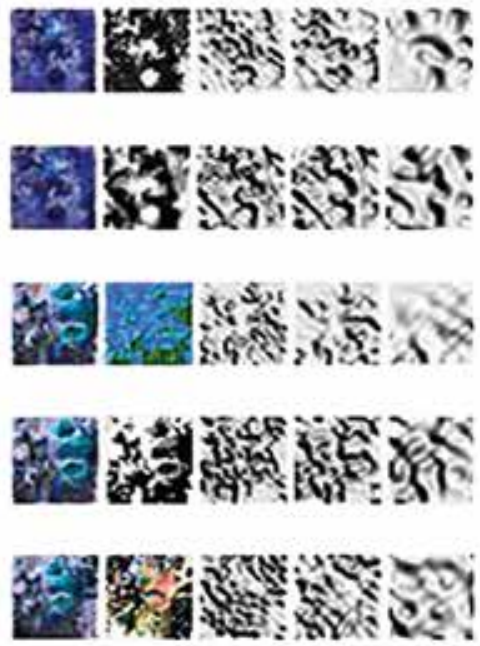

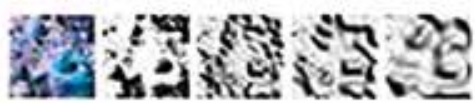
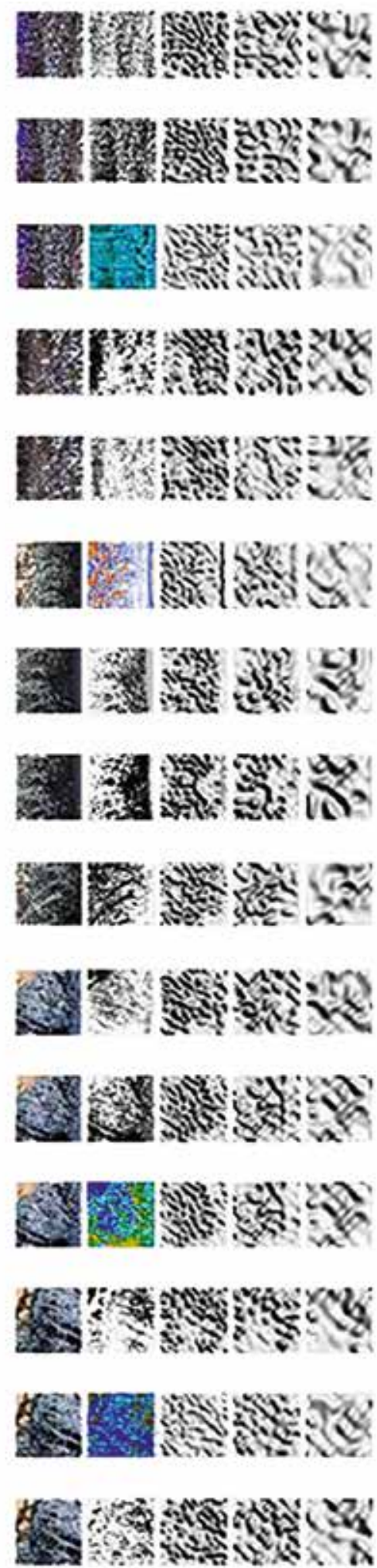

Figure 4. Image sampling to explore range of surface formal characteristics. 
dimensions in literature, this approach successfully generated a wide range of shape types to determine suitability. From these discussions, specific characteristics emerged as desirable in the final forms.

Images were then selected that generated uniform roughness across the panel with subtle variation locally. One particular image was most effective at achieving a consistent density and depth of relief that emulated an oyster reef (Fig.5, left). This image documents barnacles, oysters and other organisms in an early stage of growth across a panel of similar size to our $25 \times 25 \mathrm{~cm}$ [9.84 by $9.84 \mathrm{in}$.] test dimensions, and due to its consistency was used as background for remainder of studies.

Image sampling produced a rich variety of forms derived from images of actual habitats. However, the results lacked consistency and when modifications to the image were desirable, a significant amount of image editing became necessary, yet still without achieving any form of parametric control. As conversations on the appropriateness of the forms gave way to specific geometrical considerations, the need for a new layer that permitted control while maintaining the organic and amorphous qualities was needed. Ultimately, the image sampling procedure lacked control and consistency in the results and required the augmentation of simple overlay shapes to ensure hierarchy of habitat relief.
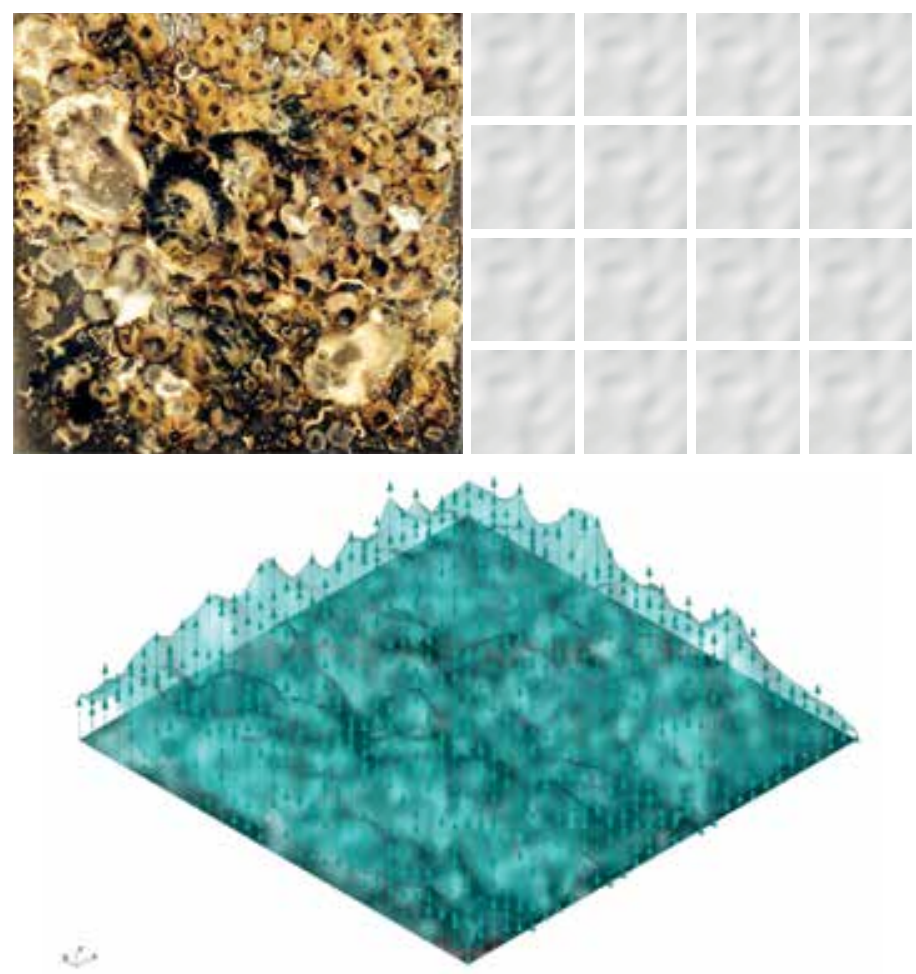

Figure 5. (upper left) Barnacle-encrusted surface selected as background image for image sampling, (upper right) range of sampled surfaces with increasing density of sample mesh points left to right (10-20-30-40) and increasing height of offset in 1,5 cm increments on y-axis top to bottom (1,5-3-4,5-6 cm) [0.59-1.18-1.77-2.36 in.], (bottom) offset of surface by vectors at mesh vertices defined by point grid at base plane. 
With the establishment of a suitable background image and sampling density, we introduced the shape overlay. We used the identified basic shapes $($ circle $=$ relief pocket, rectangle $=$ circulation shelf) to augment the background surface with specific habitat matrix as overlay in the habitat panel (Fig.6). The added forms introduced an overlay of habitat hierarchy

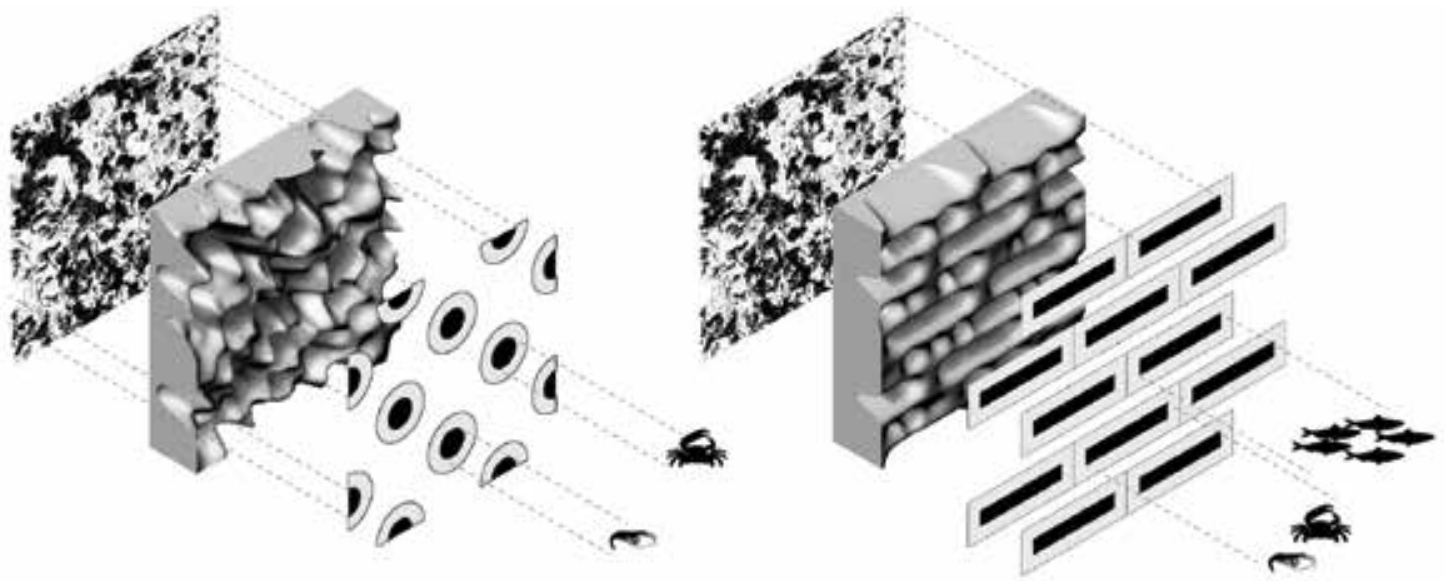

Figure 6. Effect of augmenting image with parametric shapes and the user groups primarily affected by the habitat typology.

to compliment the patterning achieved with the image sampling. In the script, the basic shapes are spliced with the image sampling to maximize or minimize the offset surface in response to circle diameter or rectangle dimension. Inner boundaries define the minimized offset, equivalent to black values in the sampled image, and outer boundaries define maximized offset, equivalent to white values in the sampled image. The combination of image sampling and shape overlay created spatial heterogeneity within the panels, which may better serve to promote diversity of occupants. The selected results of combining the pocket and shelf geometries to the image sampling are shown in Figure 7.

A pattern exhibiting attractive features was selected for fabrication, and the surface was milled in medium density fiberboard (MDF) as a pair of bookmatched tiles (Figs. 8 and 9). Twenty tiles were produced for submersion in artificial saltwater environments that varied height of relief from 0-6 cm height [0-2.36 in.]. The prototype panels were fabricated as $25 \times 25 \mathrm{~cm}$ [9.84 by 9.84 in.] panels to test geometry and fabrication techniques as full-scale surface samples. The full range of relief was CNC milled in MDF and used to cast silicone rubber molds. Shellac was applied to the milled MDF surface to seal it, and silicone casting rubber was poured over the tile. Mold Max 10 by Smooth-On was used for the silicone rubber castings of the negative. Once removed, the silicone rubber became master molds for the casting of multiple tiles in concrete. 


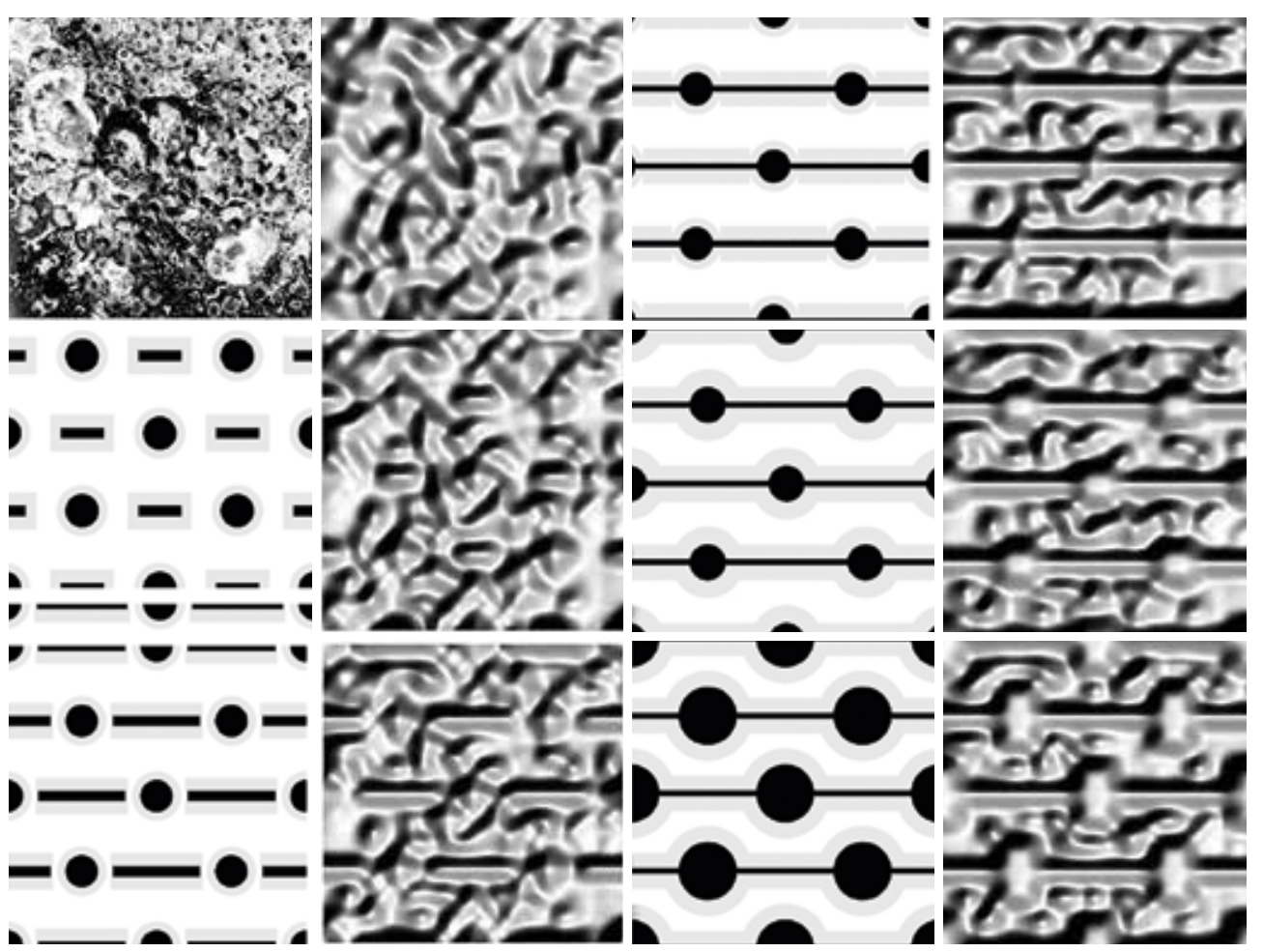

Figure 7. Increasing the dimensions of shape overlays for pronounced effect. The dimensions of each habitat shape are modified for specific evironmental conditions and creature preferences.

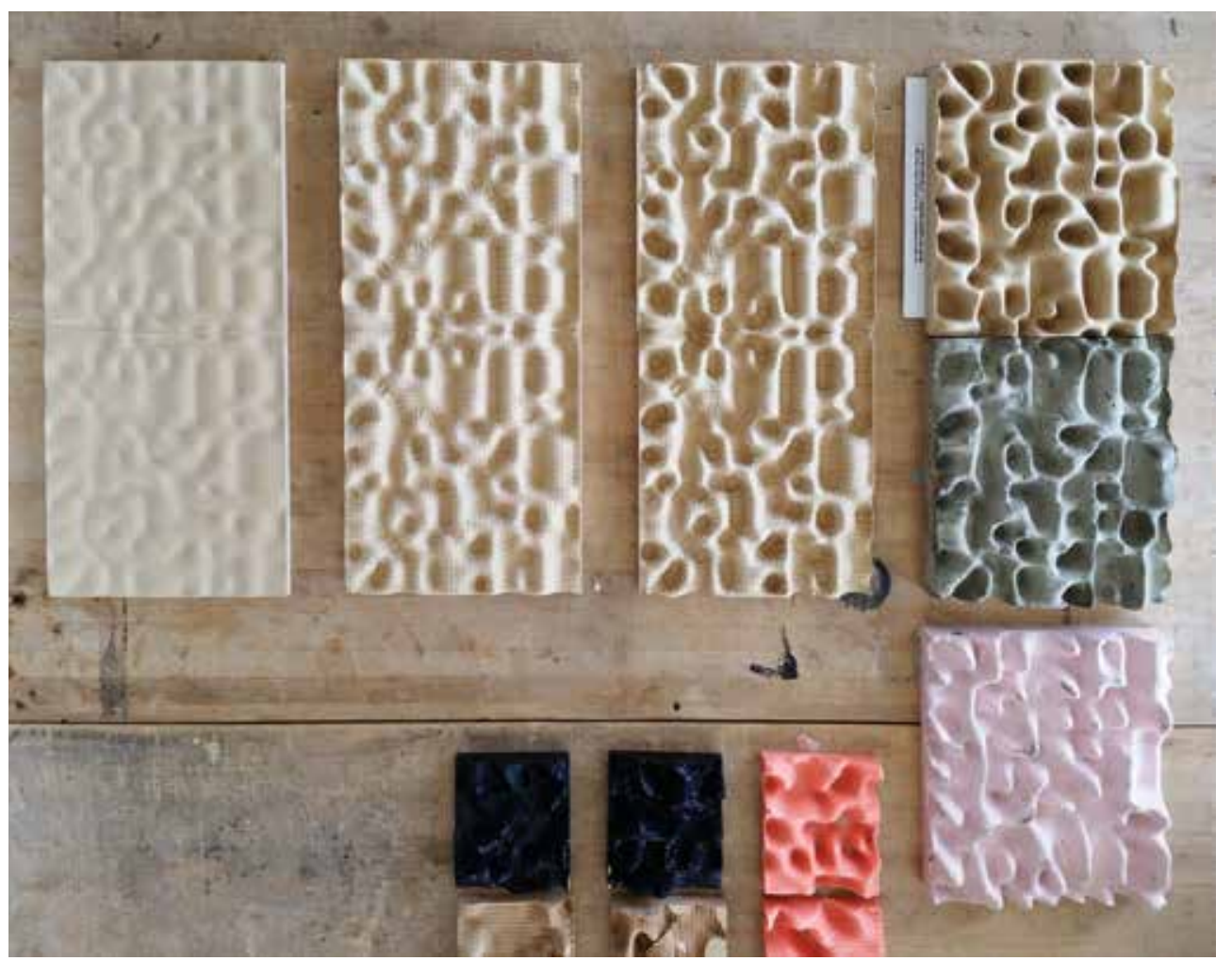

Figure 8. Final series of low to high relief panels cut for aquarium experiment - MDF, concrete and silicone panels shown at right. Additional featureless concrete panels (not shown) were cast as baseline for comparison in the study to simulate typical seawall conditions. 

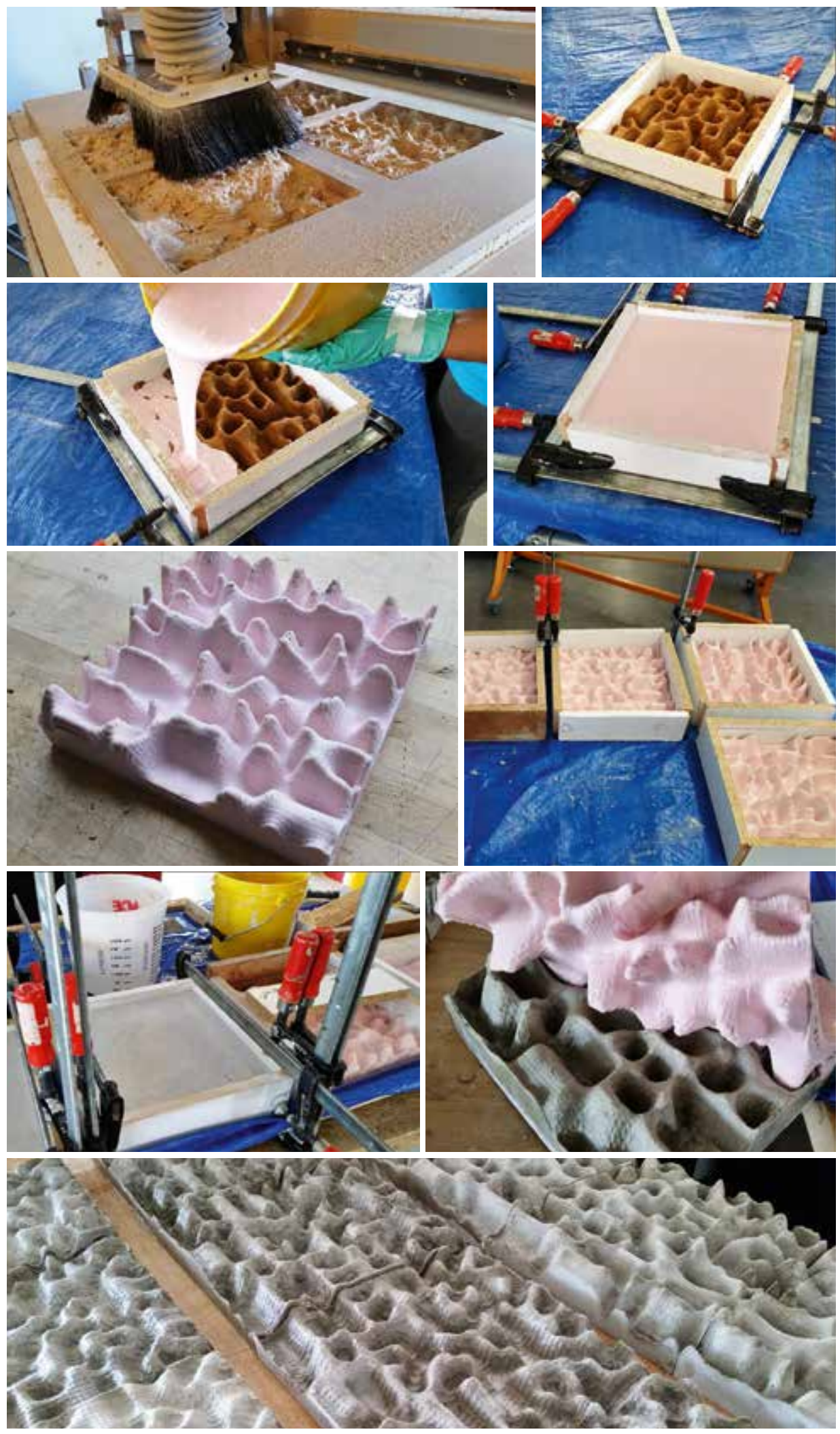

Figure 9. Fabrication workflow: Selected $25 \times 25 \mathrm{~cm}$ [9.84 by 9.84 in.] surfaces were milled in MDF and sealed with shellac (1-2), silicone rubber was poured over the MDF to create the master molds (3-5), master molds lubricated and concrete poured over (6-7), removal of complex molds from surface (8), and final sixteen tiles delivered for aquarium experiments (9). 

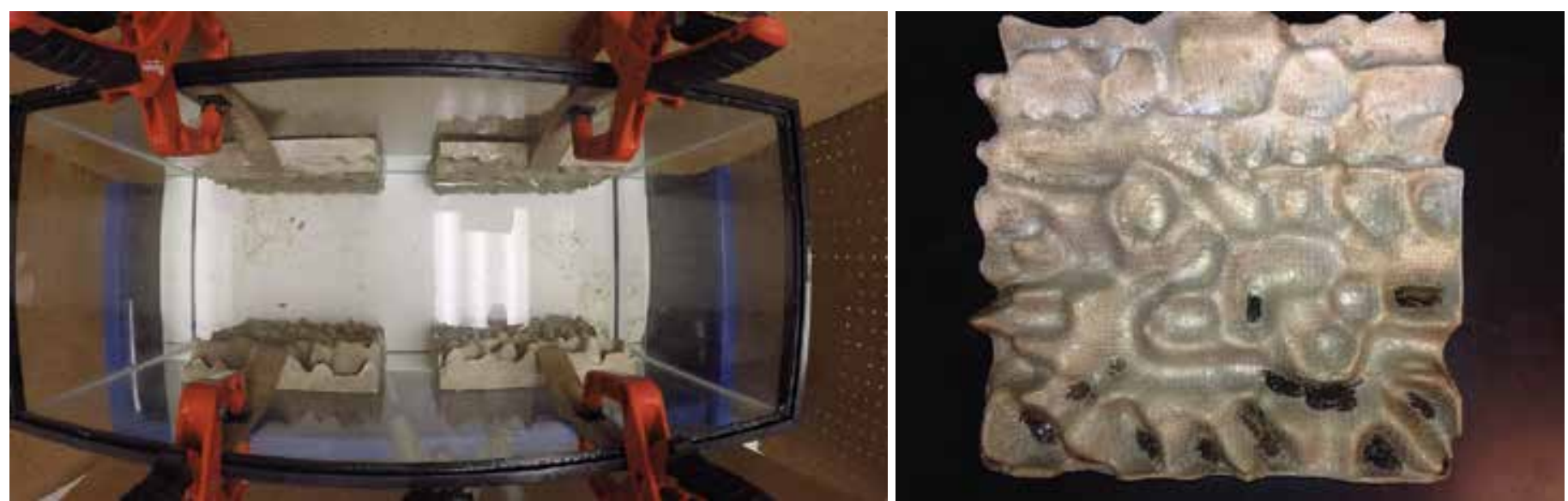

Figure 10. (left) Plan view of saltwater aquarium with panels secured by clamps, and (right) panel removed from water with black marsh crabs hiding in relief areas.

The final panels were developed for a controlled aquarium experiment to provide evidence of common species interacting with the panels. The initial experiment set up is shown in Figure 10. Panels of varying relief heights were introduced in singular and mixed arrangements. Black marsh crabs were introduced to the environments, allowed time to acclimate, and then panels were revealed. In summary, the preliminary results indicated a critical threshold for habitat preference. Low relief panels were equivalent to featureless panels in terms of crab response. In contrast, the medium and high relief panels had nearly $100 \%$ response by the crabs, indicating a tradeoff among material quantities, efficiency of producing panels and habitat values.

\section{CONCLUSION}

Our methodology for developing artificial habitat panels consisted of image sampling enhanced with a parametric model of pocket (circular) and shelf (rectangular) shapes. These basic shapes were derived from simplified versions of natural habitats and used to augment a background image with variable hierarchy of habitat structure. The shape definition is an evolving language that may form the foundation to an algorithm-based generator for habitat types. The results of this methodology included a wide range of biomorphic surfaces, of which a select group was further developed and then cast in concrete for aquarium experiments. Image sampling proved an effective way to achieve the heterogeneity of surface without existing research on the specific habitat dimension of many species. Although in our method we found distinct forms that would satisfy the conditions of an artificial reef, a more substantial photo survey of natural habitats would be ideal.

In terms of the effectiveness of the panels, our approach resulted in a highly modifiable script in Grasshopper that can accommodate any shape 
input or density to define simple relief habitat. One shortcoming of this method is the limitation of relief pockets to provide full protection.

Our future work further investigates how this approach may include more complex habitat structures to accommodate full relief.

Marine environments are complex and require feedback in the development of new technologies. In the intertidal zone, there are multiple stress gradients, which may affect the direction and strength of species interactions. Organisms try to minimize the effects of both abiotic and biotic pressure by occupying the parts of the habitats that are more suited to life/survival. The community structure along these environmental gradients is shaped in a somewhat predictable manner by dynamic interactions.

A community structure is influenced by predation, competition, biological disturbance, physical disturbances, configuration of the habitat, vertical and horizontal gradients, and the heterogeneity of the species along the environmental gradient. The interplay of abiotic and biotic factors is the controlling element of the species mosaic. The structure of the habitat affects not only the secondary foundation species themselves, but also the abundance and diversity of the assemblages. Thus, our design for the panels tries to decrease both abiotic and biotic pressure on the residents of the seawall, and proving our design can effectively perform in this manner requires testing in an actual tidal environment. As such, and following the lab experiments, a prototype seawall habitat at a location on Lemon Bay in Southwest Florida is planned. Panels will be designed for application over an existing seawall and left in place for monitoring over several years (Fig. 11).

Scant research has been done to quantify and qualify the impact of artificial structures and the ecological functions that are lost with development of the shoreline. Artificial structures that take the place of natural substrate do operate as habitat, though habitat that houses less abundant, less diverse, and a different group of species. There is a strong need to build seawalls and other artificial structure that meets both human and environmental needs.

The eventual application of this technology may be architectural, infrastructural and landscape surfaces. In this regard, high-performance passive architecture that capitalizes on climatic factors to inform building design might expand to include biodiversity in the development of surfaces and structures. Furthermore, certification systems that seek to improve the sustainability of the built environment must integrate displacement of natural habitat in the form of building footprint and its shadows as ecological factors, e.g. Living Building Challenge. Furthermore, the vertical and horizontal surfaces of structures in urban areas may offset the lost habitat of the landscape with a much greater surface area available for colonization and biodiversity. Biomimetic structures can connect fragmented habitats, which in turn connects fragmented populations 
and has an effect on larger environmental scales (Firth et al. 2013). This concept builds on what is known as "Win-win Ecology," which introduced a method to providing key habitats within urban areas to promote basic and more complex ecosystem services (Rosenzweig 2003).

Perhaps best demonstrated with the recent surge in parametricallygenerated and performance-based surfaces in buildings, architectural styles broadly considered have pivoted back towards expression of technology, culture and diversity through use of contemporary ornament (Kolarevic and Klinger 2008; Moussavi and Kubo 2006). The emulation of the environment further reflects a shifting societal awareness of the intrinsic beauty and delicacy of the planet's finite resources, even if only symbolically in buildings for most cases. Digital technologies have enabled widespread access to imagery from environmental and biological sciences and propagated greater affinity for the natural world. The architectural ornament has reemerged within a digitally-driven design process, one that has fostered the integration of natural patterns in architectural projects. New terminology is emerging to support this expanding field

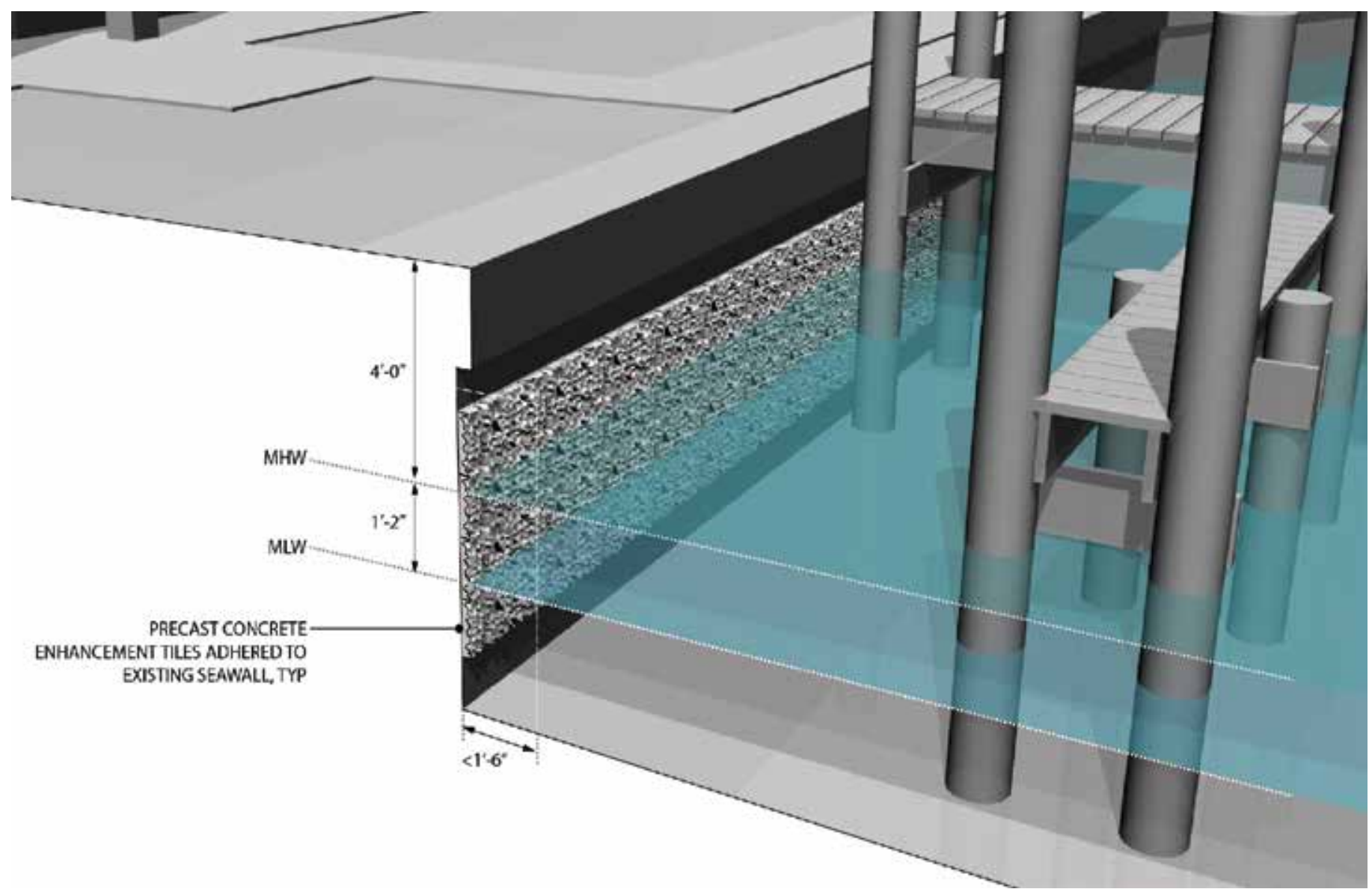

Figure 11. Schematic installation for seawall application of panels centered on tidal range in Lemon Bay in Southwest Florida, USA. 
of design inspired by imagery from the sciences - one notable example is "biornametics," a term derived from biomimetics and ornament (Gebeshuber, Gruber, and Imhof 2012). Image sampling, along with photogrammetric technologies, has enabled this "tech-transfer" from the sciences into design via photography.

City policy-makers have made strides to encourage urban ecology through native plantings, green roofs, rewilding areas, urban forestry, etc., yet the constructed elements remain passive and inert. Landscape urbanism views the city as an interconnected system of buildings, infrastructure and ecological dimensions. In order for the built environment to support this type of urban ecosystem, surfaces currently non-supportive of living systems must be activated. Ideally, these efforts strengthen human culture with materials and designs that enhance the ecology of the city.

\section{References}

Aquino-Thomas, Jessene, and Edward Proffitt. "Oysters Crassostrea Virginica on Red Mangrove Rhizophora Mangle Prop Roots: Facilitation of One Foundation Species by Another." Inter-Research Marine Ecology Progress Series 503 (2014): 177-94. doi: https://doi.org/10.3354/meps10742.

Byrnes, Jarrett E., Pamela L. Reynolds, and John J. Stachowicz. "Invasions and Extinctions Reshape Coastal Marine Food Webs." PLOS ONE 2, no.3 (2007): e295 doi: https://doi.org/10.1371/journal.pone.0000295.

Chase Anna L., Jennifer A. Dijkstra, and Larry G. Harris. "The influence of Substrate Material on Ascidian Larval Settlement." Marine Pollution Bulletin 106 (2016): 35-42. doi: http://dx.doi.org/10.1016/j.marpolbul.2016.03.049.

Ermgassen, Philine S. E. Zu, Mark D. Spalding, Brady Blake, Loren D. Coen, Brett Dumbauld, Steve Geiger, Jonathan H. Grabowski, et al. "Historical Ecology with Real Numbers: Past and Present Extent and Biomass of an Imperilled Estuarine Habitat." Proceedings of the Royal Society B (June 2012). doi: https://doi.org/10.1098/rspb.2012.0313.

Gittman, Rachel K., Steven B. Cyphers, Carter S. Smith, Isabelle P. Neylan, and Jonathan H. Grabowski. "Ecological Consequences of Shoreline Hardening: A Meta-Analysis." Bioscience 66, no. 9 (2016): 763-773. doi: https://doi.org/10.1093/biosci/biw091.

Goldman, Glenn, and Stephen Zdepski. "Image Sampling." In From Research to Practice: Mission - Method - Madness: ACADIA Conference Proceedings. Big Sky MT (USA): Montana State University, 1990: 21-28.

Harwell, Heather D., Martin H. Posey, and Troy D. Alphin. "Landscape Aspects of Oyster Reefs: Effects of Fragmentation on Habitat Utilization." Journal of Experimental Marine Biology and Ecology 409, no.1-2 (2011): 30-41. doi: https://doi.org/10.1016/j.jembe.2011.07.036.

Kolarevic, Branko, and Kevin Klinger, eds. Manufacturing Material Effects: Rethinking Design and Making in Architecture. New York: Routledge, 2008.

Layman, Craig A., Zachary R. Jud, Stephanie K. Archer, and David Riera. "Provision of Ecosystem Services by Human-Made Structures in a Highly Impacted Estuary." Environmental Research Letters 9, no.4 (2014): 044009. doi: https://doi.org/10.1088/1748-9326/9/4/044009.

Moussavi, Farshid, and Michael Kubo. The Function of Ornament. Barcelona: Actar; Cambridge MA (USA): Harvard University, Graduate School of Design, 2006.

Rosenzweig, Michael. Win-Win Ecology: How the Earth's Species Can Survive in the Midst of Human Enterprise. Oxford (UK), New York: Oxford University Press, 2003.

Sheehy, Daniel J., and Susan F. Vik. "The Role of Constructed Reefs in Non-Indigenous Species Introductions and Range Expansions." Ecological Engineering 36, no.1 (2010): 1-11. doi: https://doi.org/10.1016/j.ecoleng.2009.09.012. 
Small, Christopher. "Global Population Distribution and Urban Land Use in Geophysical Parameter Space." Earth Interactions 8, no.8 (2004):

1-18. doi: https://doi.org/10.1175/1087-3562(2004)008<0001:GPDAUL>2.0.CO;2.

"State of Florida.com I Florida Quick Facts." Accessed May 19, 2017. http://www.stateofflorida.com/facts.aspx.

Theuerkauf, Seth J., Russell P. Burke, and Romuald N. Lipcius. "Settlement, Growth, and Survival of Eastern Oysters on Alternative Reef Substrates." Journal of Shellfish Research 34 (2014): 241-250. doi: https://doi.org/10.2983/035.034.0205.

Wells, Sue, Corinna Ravilious, and Emily Corcoran. In the Front Line. Shoreline Protection and Other Ecosystem Services from Mangroves and Coral Reefs. Cambridge (UK): UNEP-WCMC, 2006. http://archive.org/details/infrontlineshore06well.

\section{Acknowledgments}

A portion of this work was supported by Florida Atlantic University Faculty Seed Grant. Special thanks to Blanca Martinez and Eric Espana for their dedication and commitment. Thanks to Dr. Evelyn Frazier and Geri Mayer for lab space and use of aquariums.

\section{Credits}

Figure 1: image courtesy of Bill Zahner of A. Zahner Co., Kansas City, USA. Figure 2: photo credit to Keith Van de Riet.

Figure 3: clockwise from top left: (1) image courtesy of U.S. Environmental Protection Agency Chesapeake Bay Program, (2) image courtesy of Dreamstime ID 72145991, user Manuel M. Almeida, (3) image courtesy of Wikipedia, user Pseudopanax at English Wikipedia, https://commons.wikimedia.org/wiki/File:Tunnelling_mud_crab.jpg, (4) image courtesy of Dreamstime ID 21537259, user Azurita.

Figures 4-7: images provided by the Authors.

Figure 8: photo credit to Pieter Conradie.

Figure 9: photo credit to Keith Van de Riet and Pieter Conradie.

Figure 10: photo credit to Jessene Aquino-Thomas.

Figure 11: image provided by the Authors.

Keith Van de Riet is an Assistant Professor at the University of Kansas (KU), School of Architecture and Design. He teaches design-build, digital design and fabrication, and architectural design with emphasis on interface between constructed and natural environments. Prior to KU, he taught at Florida Atlantic University. His research intersects design, technology and nature in the development of bioinspired systems. He received his Doctorate in Architectural Sciences from Rensselaer Polytechnic Institute with a program co-hosted by The Center for Architecture, Science and Ecology and Skidmore, Owings and Merrill in New York City. E-mail: kvdr@ku.edu

Jessene Aquino-Thomas is a marine biologist with expertise in mangrove and oyster communities in Florida. She has extensive experience in field documentation and quantitative analysis of population dynamics. Her research includes experimental work in South Florida on the impact of human and natural disturbances and on artificial habitats diversity levels in ecosystems. She is a PhD candidate at Florida Atlantic University (FAU) in Integrative Biology and has a background in biology, anthropology and sociology from FAU and Florida International University. E-mail: jaquino3@my.fau.edu

Pieter Conradie has experience working on award-winning coastal design and material development through research positions at Florida Atlantic University (FAU). Currently, he works for Brooks + Scarpa Architects in their Fort Lauderdale office, focused on a range of urban and environmental design projects. He has a B.Arch. from FAU.

E-mail: pg.conradie@gmail.com 